\title{
Polarised light stress analysis and laser scatter imaging for non-contact inspection of heat seals in food trays
}

\author{
Michael Barnes ${ }^{1}$, Mike Dudbridge ${ }^{1}$, Tom Duckett ${ }^{2}$ \\ ${ }^{1}$ Department of Food Manufacturing and Automation, University of Lincoln, Holbeach, \\ PE12 7PT, UK \\ ${ }^{2}$ School of Computer Science, University of Lincoln, Lincoln LN6 7TS, UK
}

\begin{abstract}
This paper introduces novel non-contact methods for detecting faults in heat seals of food packages. Two alternative imaging technologies are investigated; laser scatter imaging and polarised light stress images. After segmenting the seal area from the rest of the respective image, a classifier is trained to detect faults in different regions of the seal area using features extracted from the pixels in the respective region. A very large set of candidate features, based on statistical information relating to the colour and texture of each region, is first extracted. Then an adaptive boosting algorithm (AdaBoost) is used to automatically select the best features for discriminating faults from non-faults. With this approach, different features can be selected and optimised for the different imaging methods. In experiments we compare the performance of classifiers trained using features extracted from laser scatter images only, polarised light stress images only, and a combination of both image types. The results show that the polarised light and laser scatter classifiers achieved accuracies of $96 \%$ and $90 \%$, respectively, while the combination of both sensors achieved an accuracy of $95 \%$. These figures suggest that both systems have potential for commercial development.
\end{abstract}

\section{Introduction}

A large proportion of food purchased for home consumption is sold in packages. Heat-sealed packs such as the trays used in this study perform a number of functions apart from merely containing the food. One purpose is to provide a physical barrier against contaminants which might harm the quality of the food. Also food may have been packaged in a controlled atmosphere, 
such as nitrogen or carbon dioxide, intended to slow the processes that lead to food going bad.

A recent study of 105 packaging facilities at 11 factory sites (Dudbridge and Turner, 2009) concluded that in the UK alone, a potential 480,000 tonnes of food waste per year could be generated through unsound seals in food packaging. $24 \%$ of heat seals were identified in the factory audits carried out as being at risk of failure, yet only $1 \%$ of packs were identified by factory tests as being sufficiently damaged to be rejected. The majority of facilities used off-line contact methods for inspecting seal integrity, where part of the inspection mechanism must physically touch the pack. Another alternative, ultrasound, often uses water as a contact element since conventional ultrasound does not travel through the air, although advances in air-coupled ultrasound have taken place recently, with Pallav et al. (2009) reporting the ability to detect a $2 \mathrm{~mm}$ wide foreign body in cheese. In general, contact methods cannot inspect every pack on a production line at high speed, or may interfere with the sealing process or the product itself.

The objective of the research presented in this paper is to investigate noncontact methods for detecting faults in heat sealed food trays using two alternative imaging technologies. Polarised light stress images are produced by passing linear polarised light through a translucent material, which changes the polarisation of the light, resulting in coloured stress patterns when the light is passed through a second polarising filter, as seen in Figure 1. Laser scatter images are produced by measuring the amount of laser light that is deflected due to irregularities in the material being measured. For both technologies we then use conventional computer vision techniques to analyse the resulting images.

A limitation of typical machine vision system is that the set of image features for pattern recognition has to be designed by the system engineer to work with a specific configuration of product, imaging system and operating conditions. Such systems typically do not generalise well to other configurations where the required image systems may differ from those used to design the original system, or the selected features may be suboptimal for the task at hand. By contrast, this paper applies an adaptive boosting algorithm called AdaBoost (Freund and Schapire, 1999) to automatically select good features for a particular pattern recognition task. A small subset of features is selected from a very large set of candidate features, which measure statistical properties of the colour and texture distribution within the respective image region. Thus the selected features used to build the final pattern 
recognition system are optimised for the particular imaging technology under investigation.

In the experiments presented, we compare the performance of classifiers trained using features extracted from laser scatter images only, polarised light stress images only, and a combination of both image types. The results show that the polarised light and laser scatter classifiers achieved accuracies of $96 \%$ and $90 \%$, respectively, while the combination of both sensors achieved an accuracy of $95 \%$. These figures suggest that both systems have potential for commercial development.

\subsection{Related work}

\subsubsection{Seal integrity}

Sivaramakrishna et al. (2007) used an automated squeeze test system to inspect the quality of seals in brick type packages, i.e. cardboard packages used to hold liquids, which are often seen on supermarket shelves as milk or juice cartons. When the pack had been sealed but before it was fully folded into place, the system applied pressure to the pack and checked for resistance. The system took less than 30 seconds to assess each pack and detected $89 \%$ of faults of 100 micron diameter.

The use of ultrasound scanning to detect faulty food package seals is described in Raum et al. (1998). Ultrasound signals were bounced off an area of package seal, showing a difference in the amplitude (loudness) of the reflected signal at certain frequencies depending on whether the seal contained a fault or not. Adams (2000) describes a method for ultrasound scanning of medical packaging, resulting in a similar difference which he explains as being due to the inability of ultrasound to travel through air, meaning that the ultrasound transmitted into air-filled faults only passes through the first layer before bouncing back.

Morita et al. (2007) used terahertz radiation to scan package seals. Terahertz radiation, located between infrared and millimeter radiation, has advantages over light in that many materials which are opaque to the visible spectrum are only translucent with regards to the terahertz band, allowing a terahertz sensor to inspect the seals of opaque packages as though they were translucent. Water-filled channels proved harder to detect than airfilled channels, but it was shown that on a conveyor moving at $500 \mathrm{~mm} / \mathrm{s}$ the system could detect air-filled channels much smaller than 50 microns in width. 
Harper et al. (1995) describe the use of light penetration tests to inspect the thickness of the seals, whereby a translucent seal allows more light to pass through if the adhesive is not thick enough to ensure a strong seal. However this is no longer a common cause of seal faults, perhaps due to better quality control during pack manufacturing.

\subsubsection{Polarised light}

In most cases, polarised light analysis of translucent materials is done by shining a white light through a linear polarising filter, which passes through the translucent materials, then through a polarising filter positioned at an orientation of $90^{\circ}$ relative to the first filter. Alternatively, an LCD monitor can be used as a source of white polarised light (Rehorst, 2006) since the same method is used in every pixel of an LCD monitor to allow or block the light from passing through. This approach was used for the experiments described in this paper.

The use of polarised light to produce stress patterns was first described by Auguste Michel-Lévy in 1884. The colours are formed due to the birefringence of the observed material, causing the light passing through it to split into two. As shown in Figure 1, on reaching a polarising filter, only the section of the light waves aligned with the filter can pass through, which forces the light rays to recombine into a single light wave. The properties of the transparent material that the waves have passed through will cause them to become offset to one another, so recombining them will produce a new light wave with different frequency (McCrone et al., 1984).

The use of this kind of stress analysis in translucent materials is well known in other applications. A common use is in glass blowing to ensure that the newly-formed glass shape does not have obvious weak points that will be prone to breakage (Coelho et al., 2011). Following the same principle, the use of polarised lasers to analyse the strains on microcircuitry was described in Inzinga et al. (2009). Given the microscopic nature of the pieces that make up a single semiconducting component, it is necessary to observe stresses in these pieces that might shorten the working life of the semiconductor.

Gadow et al. (2009) used the term PLOTA, Polarised Light Optical Texture Analysis, to describe the combination of polarised light stress analysis with computer vision. In an example, they showed that the texture of the polarised light stress pattern on different parts of an injection-moulded gear ring was an indicator of how much strain that area could be safely put under, allowing the manufacturing process to be modified to focus the material 
strength in the areas of the gear which will be exposed to the strongest forces when in operation.

\subsubsection{Laser scatter}

Laser scatter, sometimes called laser speckle, refers to the way in which a laser can be seen to deform or spread out based on the properties of the surface it is shining onto. By measuring the amount of scatter, it is possible to determine various properties of the surface in question. For example, the amount of scatter produced by shining a laser beam onto the surface of a kiwifruit (Baranyai and Zude, 2009), or a peach (Lu and Peng, 2006), can give a good indication of the firmness of the fruit, which can provide an indication of the ripeness or quality of the fruit. Cho and Han (1999) performed similar tests on apples, using a red laser and image features relating to the colour channels of a CCD sensor to estimate the firmness of the apples.

In medicine, laser scatter has been used to identify skin affected by the disease scleroderma, since the condition causes lesions characterised by changes to the fibrous nature of the skin, which causes a laser beam to scatter more in one direction than the other. De Vries et al. (2000) took biopsies of skin with and without these lesions and took images with a laser beam being shone onto the skin. It was observed that the laser spot on lesioned skin tended to have a higher ratio between the major and minor axes than the laser spot on non-lesioned skin.

Wong et al. (1997) used laser scatter measurements to identify the level of wear on CNC machine tools. The mean and standard deviation of the intensity of a line of laser light was measured and a good correlation was found between the level of wear and the standard deviation of the laser's intensity distrubution.

Figure 2 shows photographs of a laser pointer shone through a good pack seal and a faulty pack seal. The laser spot from the pointer visibly scatters further in all directions in the presence of a fault. This is a typical scatter setup, where a narrow beam of laser light is shone on or through an object and the laser dot is photographed. As an alternative, the laser beam can be spread into a line by a shaped lens, allowing it to shine onto a whole row of a pack at a time. This has limitations in only being able to observe scatter in one direction but it can scan the entire width of a pack per frame. Figure 3 shows this kind of laser beam in operation. 


\section{Materials and methods}

\subsection{Pack sealing and fault production}

The experiments were conducted using a set of transparent rigid plastic trays, of the type shown in Figure 4, which were sealed with transparent flexible film using an Ishida tray sealing machine shown in Figure 5. These tray sealers work by pressing the film against the tray and applying a high temperature, which melts together a coating on the underside of the film and the top of the tray, producing an airtight bond.

The most common cause of seal faults is contamination of the area between the tray and film, so to produce artificial faults we used copper wire to contaminate this area. In previous experiments we attempted to use human hair but that required adhesive to be used and there were concerns that the adhesive might be detected by the sensor. The wire was temporarily fixed to the outside of each pack using poster putty and bent across the seal area. Effort was made to ensure that the position and orientation of the wire was randomly distributed, which was aided by a tendency of the wires to shift during the sealing process.

Faults were produced using 125 micron copper wire, which was only attached to the outside of the pack to make it easier to remove the whole wire. This also allowed all trace of the wire to be removed, avoiding the risk of marking the inside of the pack in a way that might affect the classifier. Tungsten or molybdenum wire could be used for future tests to allow the production of smaller faults. After the faults had been produced, all of the trays were carefully inspected and 3 trays were removed from the original set of 120 trays because additional unintended faults were observed in the seals of these trays, leaving an experimental data set of 117 trays (60 containing faults, and 57 containing no faults).

\subsection{Image acquisition}

Two methods were used to capture images of the packs: polarised light and laser scatter imaging, as described in the following paragraphs. All images were recorded in the dark to reduce interference from ambient light.

Polarised white light, when passed through a translucent object then through a linear polarising filter, takes on a coloured pattern based on the properties of the object it has passed through. The colours tend to vary in regions where a seal is improperly formed. Figure 6 shows an example with 
four clearly visible faults. This image was taken with a camera and a homemade polariscope constructed from an LCD monitor shown in Figure 7. An LCD monitor showing a white screen was laid flat on a table. A Sony Alpha 200 digital SLR camera was fixed to a stand and positioned looking directly down at the monitor. A polarising filter was then positioned on the camera to block the light from the monitor. The polarising filters on the monitor and on the camera then take the role of the two polarising filters shown in Figure 1.

Laser scatter imaging involves recording a sequence of images of a moving object as it passes under a laser line. The system we employed, built into the SICK Ranger camera, produces two channels of intensity data. The first, known as the direct channel, consists of the intensity of the laser line at its centre, while the second consists of the intensity of the laser at a point beyond its normal area. This second channel, known as the scatter channel, indicates how the laser light is being scattered, as shown in Figure 8. This output resembles the method used by a line-scan camera but using a multiple line sensor, sometimes referred to as band-scan. The camera setup used to take these images is shown in Figure 3. Figure 6 also shows the pack seal in the scatter and direct channels. Three of the four seal faults are immediately obvious as bright patches, with the fourth being detectable by the software. The bright area flare close to the middle of the seal is a common feature, allowing the classifier to look for anomalies in its form to detect whether there is a fault in that location.

\subsection{Image pre-processing}

The first stage of image pre-processing was to locate the seal on each pack. Using polarised light images it is easy to segment the box from the background, since the background is black due to being blocked by the polarising filter. A template-fitting approach was then applied. The angle of the pack was measured by locating the centre of the pack, then the pack image was rotated to position the pack horizontally. The lengths of both axes of the pack were then measured. The template was then scaled to the same size as the pack, rotated to the same angle as the pack in the original image and positioned so that it shared a centroid with the pack in the image.

For laser scatter images this approach proved unreliable since the edge was not always visible. Manual markup was therefore used to indicate to the system where the seal was located. In an industrial application, this problem 
could be solved by placing a guide rail on either side of the conveyor belt to ensure that each pack passes in a known position and orientation.

Once the seal had been located, the seal area on the side of the packs investigated was subdivided into 16 regions as shown in Figure 9, where each region was approximately $1 / 20$ of the length of the pack. For the subsequent analysis, a separate classifier was trained to detect faults for each of the affected regions, as described in the following sections.

If a pack were to be deformed then the seal might not be in the location specified by the template. This would be likely to produce anomalies in the laser scatter image at the areas around the deformity. Under polarised light, the deformity could be detected since the area around the pack is black, allowing the shape to be examined. However, for the purposes of the "proof of concept" study presented in this paper, we did not investigate the issue of deformed packs.

\subsection{Feature extraction}

The next step of image processing is to extract image features that should help to indicate the presence or absence of faults in a given image region. As described in Section 2.3, the pixels that make up the seal are known and divided into regions of interest. A number of different filters are applied to the channels of the original images, described in the following subsections, and then the results are summarised by region to provide the input features for classification with the machine learning algorithm described in Section 2.5. The pipeline of processing steps involved in feature extraction is described as follows.

\subsubsection{Input channels}

First the respective image types are separated into channels, with each channel comprising a 2-D array with the same resolution as the input image. For polarised light images, there are four channels: red $(R)$, green $(G)$, blue $(B)$ and intensity $\left(I=\frac{1}{3}(R+G+B)\right)$. For laser scatter images, there are two channels: scatter $(S)$ and direct $(D)$.

\subsubsection{Gradient and range filtering}

In this step, the input channels described above are passed through a number of different filters in parallel: range, horizonal gradient, vertical gradient and non-directional gradient, described as follows. The output of this step includes the original 6 channels plus the four filtered versions of these 6 
channels. This has the effect of increasing the number of channels for subsequent processsing by a factor of 5 , producing 20 channels of data for polarised light images and 10 channels for laser scatter images.

Range filter. The range statistic describes the difference between the highest and lowest pixel values in a $3 \times 3$ area centered on the pixel of interest. The result is the highest pixel value minus the lowest pixel value.

Gradient filters. The Sobel filter, as used in our experiments, is a discrete differentiation operator, which computes an approximation of the gradient of the image intensity function at a given point. This is known as a sharpening filter since it will tend to highlight lines and edges in images.

The gradient formulas for horizontal and vertical gradients, respectively, can be represented as:

$$
G_{x}=\left[\begin{array}{lll}
-1 & 0 & +1 \\
-2 & 0 & +2 \\
-1 & 0 & +1
\end{array}\right] * A, \quad G_{y}=\left[\begin{array}{ccc}
-1 & -2 & -1 \\
0 & 0 & 0 \\
+1 & +2 & +1
\end{array}\right] * A
$$

where $*$ is the convolution operator and $A$ is the matrix being processed, in this case one channel (e.g. colour channel) of a given image. A non-directional representation of the gradient can also be produced by adding together the absolute values of both directional gradients $G=\left|G_{x}\right|+\left|G_{y}\right|$.

\subsubsection{Entropy filtering}

In this step, an entropy filter is applied to all of the channels obtained from the previous step, described as follows. The output of this step includes the unfiltered versions plus the entropy-filtered versions of these channels. This has the effect of increasing the number of channels for subsequent processsing by a factor of 2 , producing 40 channels of data for polarised light images and 20 channels for laser scatter images.

The entropy of a set of image pixels refers to an estimate of their information content based on the relative frequency of occurrence of the grey levels in the corresponding image region. The entropy filter ignores the spatial layout of the pixels and instead considers the distribution of grey levels, in our case those in a $9 \times 9$ grid centered on the pixel of interest. The entropy of a set of values is calculated by taking a histogram of all the values presented and then normalising the histogram so that the total of all bins equals 1 , giving a probability distribution. The entropy is then given as $-\sum_{i}\left(p_{i} \log _{2}\left(p_{i}\right)\right)$, where $p_{i}$ is the probability of occurrence for grey level $i$. 


\subsubsection{Additional virtual channels for polarised light images}

While the steps described above are applied to both polarised light and laser scatter images, the polarised light images offer the opportunity to provide additional features relating particularly to the colour channels.

In initial experiments it was found that where a particular statistic had been calculated for the red, green and blue channels separately, it could also be advantageous to include the minimum, maximum and range (i.e. maximum minus minimum) of the three values $(R, G$ and $B$ ) to provide additional candidate features for possible selection by the machine learning classifier (AdaBoost). So, for example, the "minimum range" statistic for a particular pixel would be calculated as the minimum of the red, green and blue range-filtered values for that pixel. This approach was found to be particularly useful when using the minimum entropy for the three colour channels, because a fault tended to be characterised by a spike in the entropy of all three colour channels, while a spike in one or two of the colour channels was not uncommon but tended not to represent a fault.

This step has the effect of adding an additional $3 \times 10=30$ channels for the polarised light images, producing 70 channels of data for polarised light images (including the 40 channels from the previous step), while the number of channels (20) remains unaffected for the laser scatter images.

\subsubsection{Regional summary features}

Finally, the above filtered data, comprising 70 channels (images) for polarised light images and 20 channels (images) for laser scatter images, are summarised statistically by region, according to the image regions defined in Section 2.3 and Fig. 9. Six different region summary statistics are calculated per channel, namely the mean, variance, skewness, minimum value, maximum value, and range (maximum minus minimum) value of all the pixel values in the corresponding region. This step has the effect of producing six output features for each of the channels, producing a total of 420 features per region for polarised light images and 120 features per region for laser scatter images.

In general, one could add as many features as desired to improve the performance of the trained classifier, because the approach used for classi-

fication, described in the following section, includes automatic selection of the most useful features for a particular pattern recognition task from a very large set of candidate features. Once the classifier has been trained, only the 
selected features need to be calculated when the trained classifier is deployed in the intended application.

\subsection{Classification}

The AdaBoost algorithm (Freund and Schapire, 1999) is used to build a classifier, which combines results from so-called "weak" classifiers into one "strong" classifier that performs better than any of the weak classifiers alone. In our case, each of the weak classifiers consists of a decision stump, or onelevel decision tree, constructed from a single image feature. For each decision stump, a threshold feature value is selected, and the stump contains two leaves, one for values below and one for values above the threshold.

The high performance of the final strong classifier is due to the emphasis put on the training examples which are most difficult to classify during the learning process. This method is called boosting. During training AdaBoost makes a number of iterations through the training data. Each time it finds the next best image feature to improve the number of correctly classified examples, prioritising those examples which were misclassified previously. In each iteration one feature is selected and assigned a weight and a threshold to create a new weak classifier. The weak classifiers are then combined into a strong classifier, where each weak classifier has a weighted vote in the classification of a given example.

The Real AdaBoost algorithm proposed by Schapire and Singer (1999) is a generalisation of this algorithm that provides a lower error rate by allowing weak classifiers to vote by their individual degree of certainty instead of simply voting yes or no. In our MATLAB implementation we used the Real AdaBoost implementation within the GML AdaBoost Toolbox (Vezhnevets, 2006) to select weak classifiers from a set of every possible threshold of every individual region feature to produce a strong classifier.

In our system the Real AdaBoost algorithm was used to classify individual regions of seal images into two categories: regions with faults and regions without faults, based on the features defined in Section 2.4. The reduced set of selected weak classifiers allows for preprocessing only the most useful features in the final trained system, saving considerable computation time. 


\section{Results and discussion}

\subsection{Training and testing}

The data set used in these experiments consisted of 117 translucent plastic trays, sealed in the Ishida heat-sealing machine as described above. 60 packs were faulty, with up to 5 faults per pack, and 57 packs contained no faults. Faults were introduced into one side of each pack only. The following experiments were carried out on a hold-one-out regime, with each one of the 117 packs being tested using a system trained with the other 116 packs. This approach allowed us to work with a relatively small data set and still make use of a relatively large amount of training data.

Ground truth data was produced by taking a greyscale image of each tray under polarised light, then manually drawing in faults in colour. In most cases the faults were visible in the greyscale images while in others it was necessary to measure the location on the pack and mark the location on the image accordingly.

As described in Section 2.3, the seal area on the side of the packs investigated was subdivided into 16 regions as shown in Figure 9. Out of these 16 regions, a total of 13 regions contained at least one fault in the packs in the database (the other 3 regions contained no faults in any of the packs). A separate classifier was trained to detect faults for each of these 13 regions, i.e. to declare whether the region contains a fault or not. For each of these regions, the corresponding ground truth information (recording whether or not the region contained a fault in reality) was obtained by reference to the manually recorded data, with a region being classified as faulty if it contained at least one fault.

\subsection{System performance}

The output of the classifier for each inspected region of the seal is a binary value, which indicates whether the seal region is considered to contain a fault (positive) or no faults (negative). By comparing these outputs with the presence or absence of faults in the ground truth data, we calculated the following statistics:

- TP - true positive, the number of regions that were classified as faulty and matched ground truth;

- FP - false positive, the number of regions that were classified as faulty but did not match ground truth; 
- TN - true negative, the number of regions that were classified as nonfaulty and matched ground truth;

- FN - false negative, the number of regions that were classified as nonfaulty but did not match ground truth.

From these statistics we calculated the following metrics:

- sensitivity $=\frac{T P}{T P+F N}$;

- specificity $=\frac{T N}{T N+F P}$.

- accuracy $=\frac{T P+T N}{T P+T N+F P+F N}$

As shown in Table 5, the classifier achieved an accuracy of $96 \%$ when trained using polarised light, $90 \%$ when trained using laser scatter data and 95\% when trained using both sensors. Adding the laser scatter data to the polarised light training data resulted in a minor reduction in the sensitivity of the classifier.

The current implementation of the system is in MATLAB running on a 2.8 $\mathrm{GHz}$ processor. For the 13 classifiers trained on polarised light images, feature extraction for the seal area took 83.4 seconds, with classification taking a further 0.009 seconds. For the 13 classifiers trained on laser scatter images, feature extraction for the seal area took 29.0 seconds, with classification taking a further 0.014 seconds. After training, only a relatively small number of features need to be calculated for the selected weak classifiers, due to the feature selection using AdaBoost, saving considerable processing time. This code has not been optimised, and much faster processing times could be expected with a compiled programming language such as $\mathrm{C}++$ for real-time applications.

The polarised light methods used here assume a translucent pack for the light to pass through, however the same techniques can be used with reflected polarised light, as seen in Figure 10. For industrial application this would probably use a specialised multiple-polarisation camera (FluxData Inc., 2011).

\subsection{Preferred features}

A summary of the features selected for classification is shown in Tables 1 and 2. This example was trained on all 117 packs although for the holdone-out experiments only 116 packs were used at a time. The differences in 
selected features were minor. Most notable for polarised light based classification is the prevalence of features based on the entropy of either raw colours, gradient or range features. The most common feature type selected is the entropy of the range, i.e. the distribution of the highest and lowest values in the $3 \times 3$ square centered on each pixel. The most commonly selected region summary statistic is the variance. Of the statistics which are not statistical moments, the maximum is selected most often, which is likely to be because faults are quite small but represent significant spikes in the input data compared to smooth seal areas. With laser scatter images, the most commonly chosen feature types are those relating to gradients or ranges rather than entropy. The mean is chosen more often than any other statistic, and the direct channel is preferred in 27 cases compared to 17 features for the scatter channel.

When using polarised light, the system required a total of 15 selected features (out of 420 candidate features) to obtain the highest overall accuracy of the image types investigated (see Table 3 for the full list of features selected). When the polarised light and laser scatter features were used together, the system required a total of 16 features (out of 540 candidate features) to achieve a similar level of accuracy (see Table 4 for the full list of features selected). Laser scatter required 45 features (out of 120 candidate features) to obtain a lower accuracy (table omitted for brevity). Here, the entropy of the gradient and range features within the polarised light image proved to be important. In contrast with using laser scatter images alone, when combined with polarised light the direct channel was used equally with the scatter channel. This is probably because the direct channel is closer to being a simple camera, which would be similar to but simpler than a polarised light image.

When using polarised light, the classifier often chose features relating to the minimum, maximum or range of the three colour channels, as described in Section 2.4.4, rather than to the individual colour channels. Some classifiers were able to produce adequate results using only one or two polarised light features, due to the uniformity of those particular seal regions. This was more common in regions toward the left end of the seal as viewed in Figure 6, which might be due to the differences in how the film is cut toward the pull-tab at the right end. No correlation was observed between the number of faults in a region and the number of weak classifiers selected for that region. Using laser scatter features tends to also require more features for classifiers toward the right of the seal, though still requiring more features throughout. 


\section{Conclusions and further work}

Polarised light imaging and laser scatter imaging both offer methods for inspecting heat-sealed packages, achieving overall accuracy rates of $96 \%$ and 90\%, respectively, when detecting faults in a database of 117 translucent tray seals. In theory these sensors can both be applied to other types of heat-sealed tray packs, including those which are not entirely composed of transparent materials. Further work should include non-clear trays, smaller faults (Raum et al. (1998) cite evidence that bacteria can pass through faults of no less than 33 microns in diameter) and testing with real-world pack faults in an industrial environment.

Overall, polarised light and laser scatter techniques show good promise for development of a commercially viable, non-contact seal inspection method which could eventually be implemented in the majority of tray packing facilities to reduce worldwide food waste from package seal faults, estimated at 480,000 tonnes of food waste, or around $8 \mathrm{~kg}$ for every citizen, in the UK alone.

\section{Acknowledgements}

This research was funded by DEFRA, the Department of Food and Rural Affairs, project code AFM284, Monitoring of heat sealed packs using thermal and multispectral imaging - a pilot study. The tray sealer was provided by Ishida Ltd. at the National Center for Food Manufacturing. We would also like to thank Prof. Nigel Allinson at the University of Lincoln for advice on possible imaging technologies for use in this study.

Adams, T., 2000. Non-destructive acoustic micro imaging of package seals. Sealing Technology 2000 (76), 7-9.

Baranyai, L., Zude, M., 2009. Analysis of laser light propagation in kiwifruit using backscattering imaging and monte carlo simulation. Computers and Electronics in Agriculture 69 (1), 33 - 39.

Cho, Y.-J., Han, Y. J., 1999. Nondestructive characterization of apple firmness by quantitation of laser scatter. Journal of Texture Studies 30 (6), 625-638.

Coelho, J. M. P., Silva, C., Almeida, T., 2011. Stress analysis in glass artwork. International Journal of Optics 2011, 7. 
De Vries, H. J. C., Enomoto, D. N. H., van Marle, J., van Zuijlen, P. P. M., Mekkes, J. R., Bos, J. D., 2000. Dermal organization in scleroderma: The fast Fourier transform and the laser scatter method objectify fibrosis in nonlesional as well as lesional skin. Labouratory Investigation 80 (8), 12811289 .

Dudbridge, M., Turner, R., 2009. Seal integrity and the impact on food waste. Tech. rep., UK Waste and Resources Action Programme (WRAP). URL http : //www . wrap.org.uk/retail_supply_chain/research_tools/research/ report_seal.html

FluxData Inc., 2 2011. Fluxdata sales literature. Website accessed 2011. URL http://www.fluxdata.com/wp-content/uploads/FD-1665-brochure.pdf

Freund, Y., Schapire, R., September 1999. A short introduction to boosting. Journal of Japanese Society for Artificial Intelligence 14 (5), 771 - 780.

Gadow, R., Kern, F., Wenzelburger, M., Rauch, J., 2009. Engineering of high performance injection moulded ceramic components by polarised light optical texture analysis. International Journal of Materials and Product Technology 35 (3-4), 324-333.

Harper, C. L., Blakistone, B. A., Litchfield, J. B., Morris, S. A., 1995. Developments in food packaging integrity testing. Trends in Food Science \& Technology 6 (10), 336-340.

Inzinga, R., Horn, G., Johnson, H., June 2009. Experimental stress mapping of etched cavity semiconductor devices. In: Proceedings of the Society for Experimental Mechanics Annual Conference. Alberquerque, New Mexico, USA.

Lu, R., Peng, Y., 2006. Hyperspectral scattering for assessing peach fruit firmness. Biosystems Engineering 93 (2), 161 - 171.

McCrone, W. C., McCrone, L. B., Delly, J. G., 1984. Polarized Light Microscopy. Ann Arbor.

Morita, Y., Dobroiu, A., Otani, C., Kawase, K., 2007. Realtime terahertz diagnostics for detecting microleak defects in the seals of flexible plastic packaging. Journal of Advanced Mechanical Design, Systems, and Manufacturing 1 (3), 338-345. 
Pallav, P., Hutchins, D., Gan, T., 2009. Air-coupled ultrasonic evaluation of food materials. Ultrasonics 49 (2), $244-253$.

Raum, K., Ozguler, A., Morris, S. A., William D. O'Brien, J., 1998. Channel defect detection in food packages using integrated backscatter ultrasound imaging. IEEE Transactions on Ultrasonics, Ferroelectrics, and Frequency Control 45 (1), 30-40.

Rehorst, M., 7 2006. LCD monitor as a polarimetric stress viewer. Website accessed 2011.

URL http://mark.rehorst.com/Polarimeter/index.html

Schapire, R., Singer, Y., December 1999. Improved boosting algorithms using confidence-rated predictions. Machine Learning 37 (3), 297-336.

Sivaramakrishna, V., Raspante, F., Palaniappan, S., Pascall, M. A., 2007. Development of a timesaving leak detection method for brick-type packages. Journal of Food Engineering 82 (3), 324-332.

Vezhnevets, A., 2006. GML AdaBoost MATLAB Toolbox. Website accessed 2009.

URL http://research.graphicon.ru

Wong, Y., Nee, A., Li, X., Reisdorf, C., 1997. Tool condition monitoring using laser scatter pattern. Journal of Materials Processing Technology 63 (1-3), 205-201. 


\section{Figures and tables}

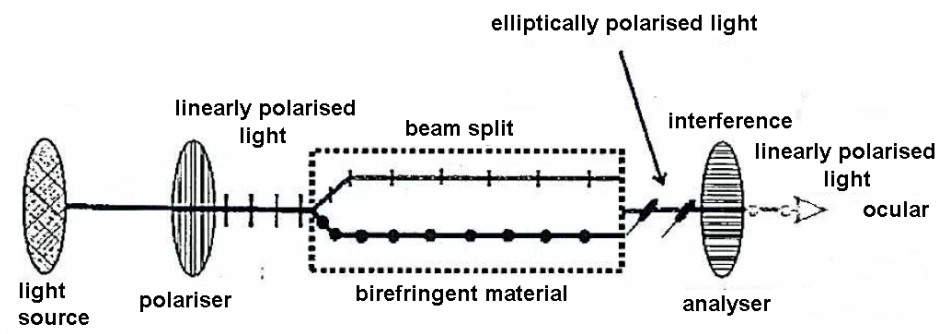

Figure 1: Demonstration of the process by which white polarised light changes to coloured polarised light when producing a stress pattern.

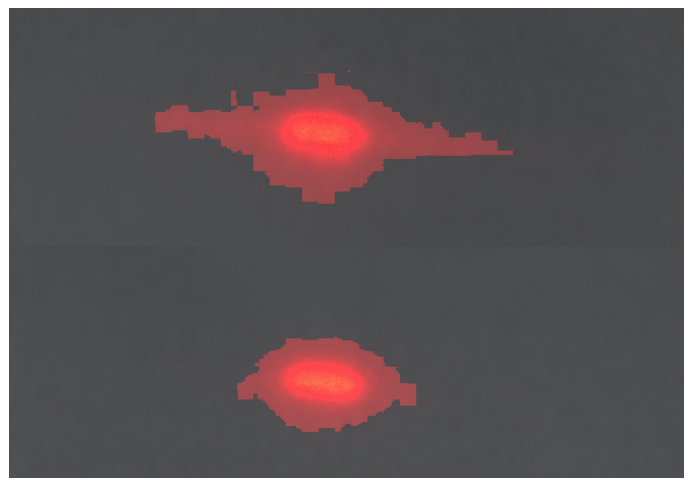

Figure 2: A laser pointer shone through a seal fault (top) and a good seal (bottom) this simple test shows that there is a potential for scatter features to determine faulty seals from good seals. The fault increases scatter in all directions but most significantly perpendicular to the fault. Non-red pixels have been darkened for emphasis. 


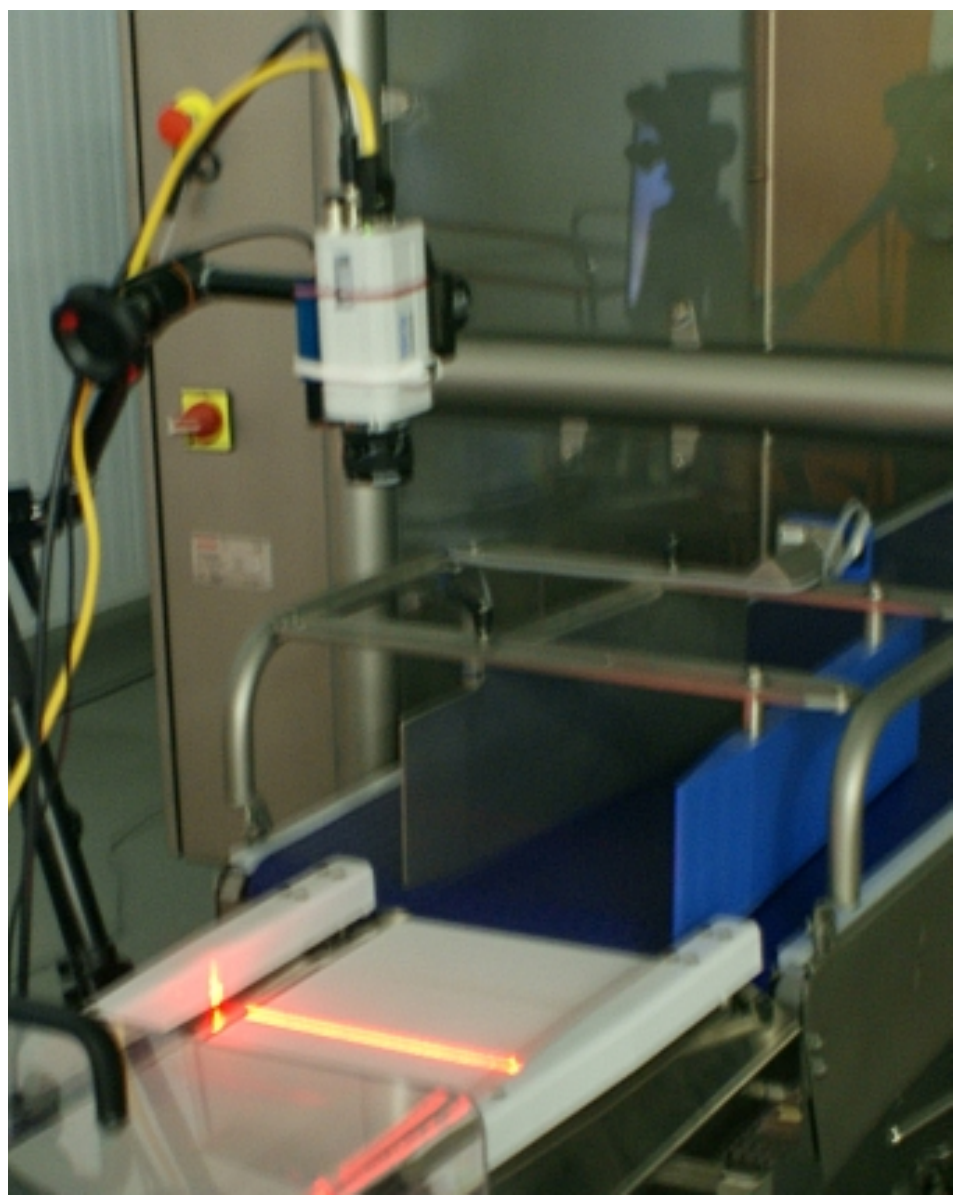

Figure 3: The laser scatter camera used in these experiments, a SICK Ranger E with an Invisio $100 \mathrm{~mW}$ laser. 


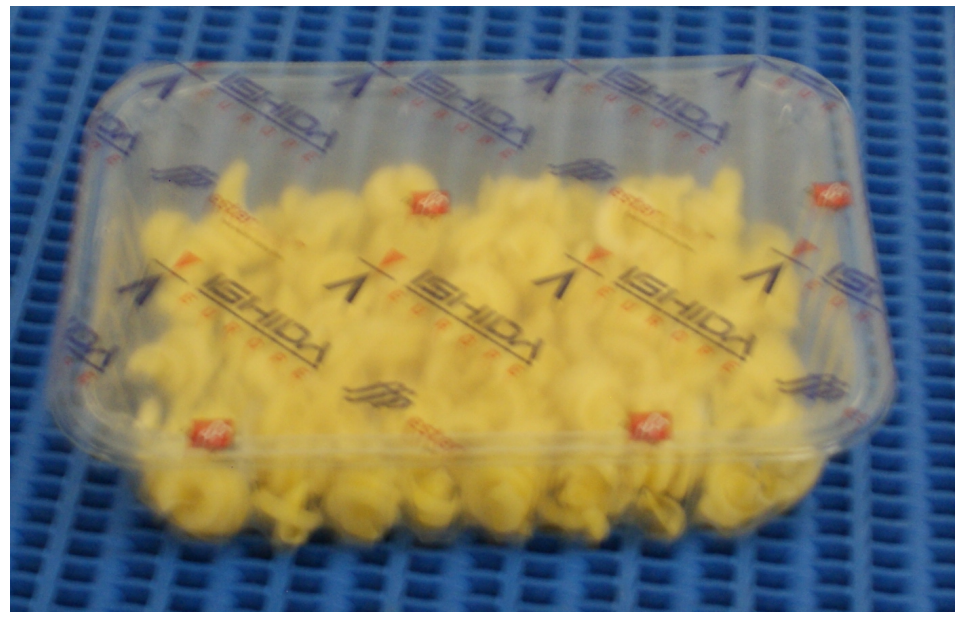

Figure 4: The tray type used in these experiments; this one contains pasta while the ones in the experiments were empty.

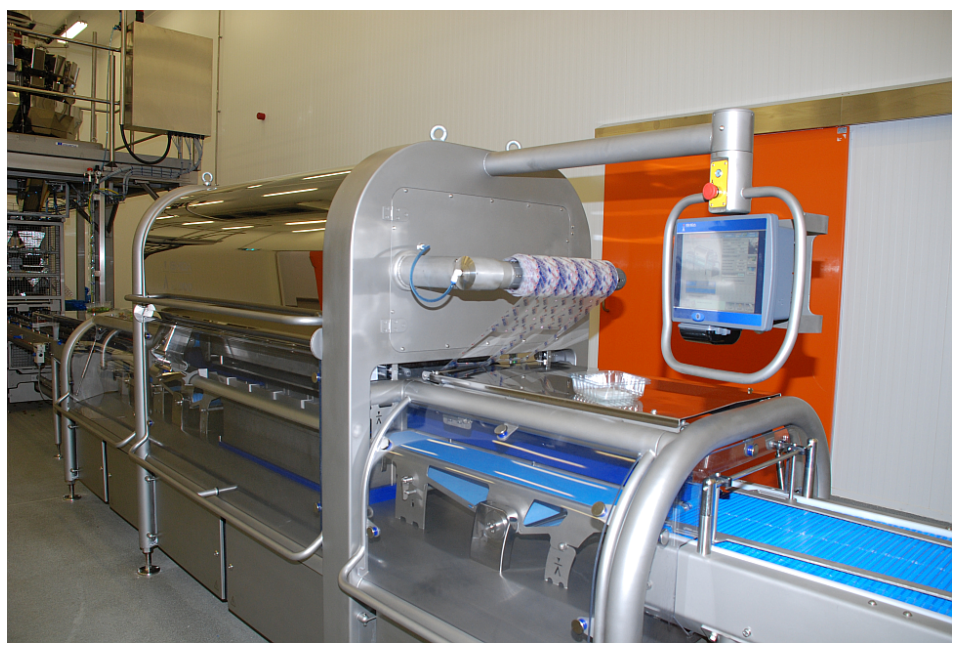

Figure 5: The Ishida heat sealing machine used in these experiments. 


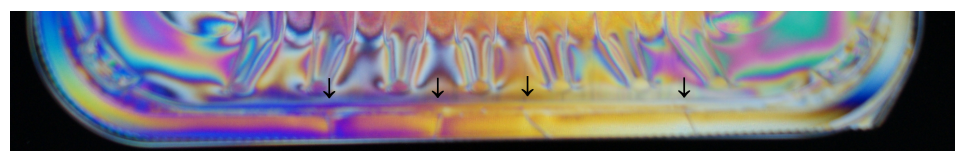

(a) Stress image

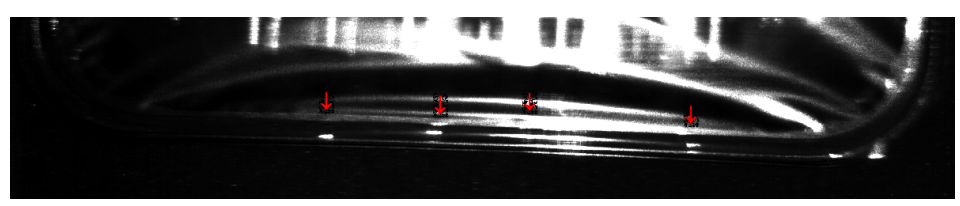

(b) Laser scatter

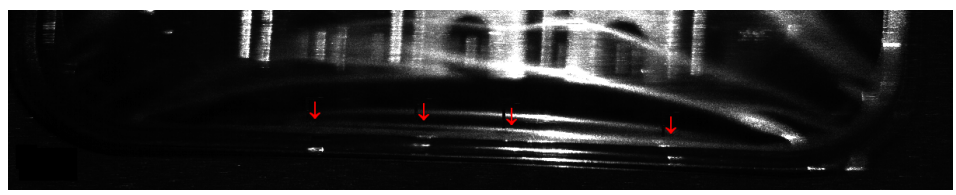

(c) Laser direct

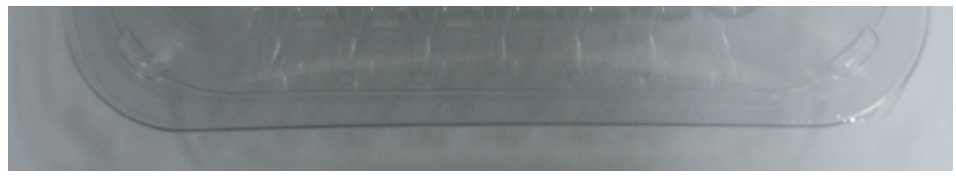

(d) Ambient light

Figure 6: A stress image, laser scatter image, laser direct image and image under ambient light of a pack seal. Arrows have been added to the stress and laser images to indicate the presence of four faults in the seal, which are not visible in the ambient light image. 


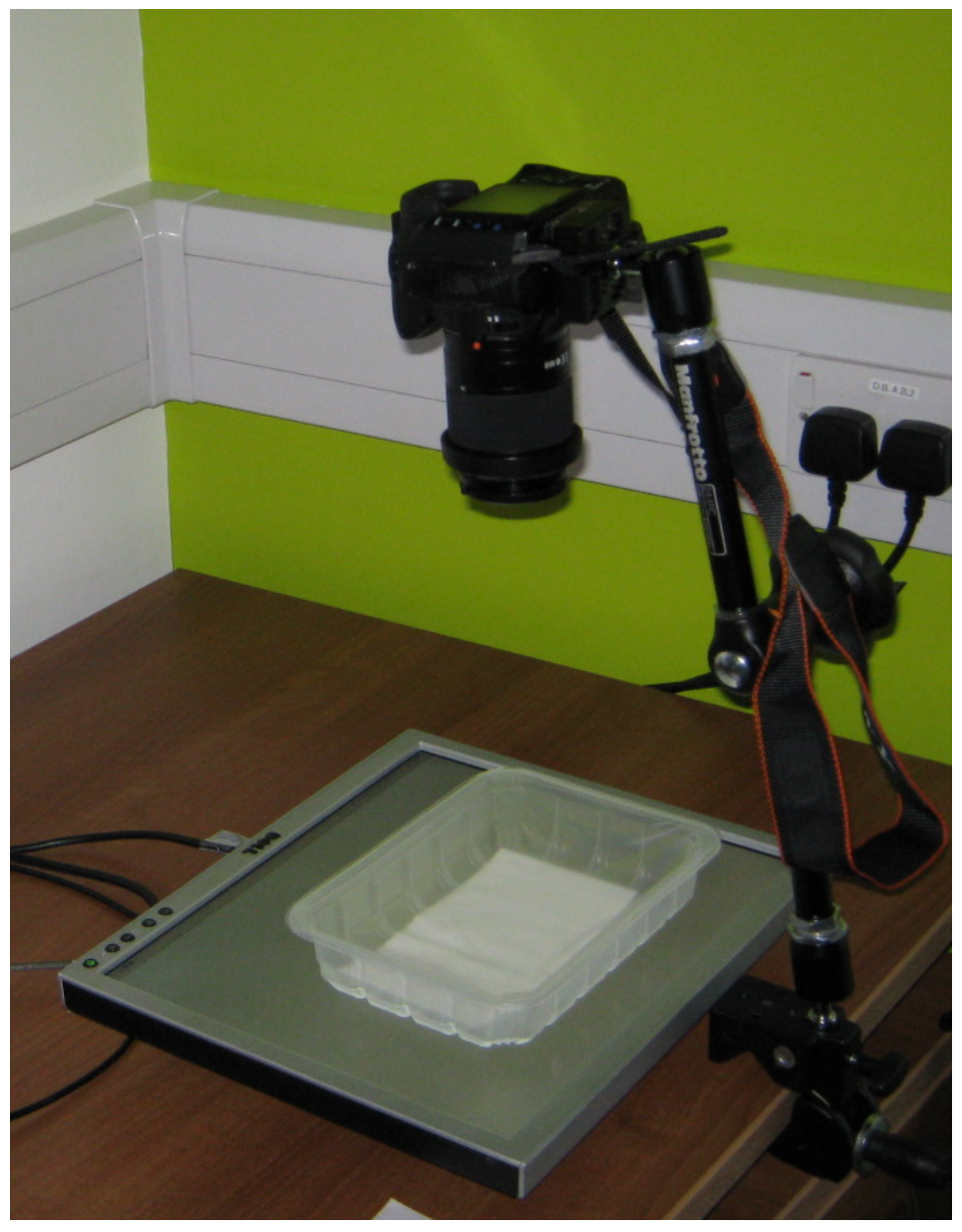

Figure 7: The makeshift polariscope used to make the polarised light stress patterns, comprising an LCD monitor and a CCD camera with a linear polarising filter at $90^{\circ}$ to the polarisation of the light coming from the LCD. The item to be inspected is placed between the two. 

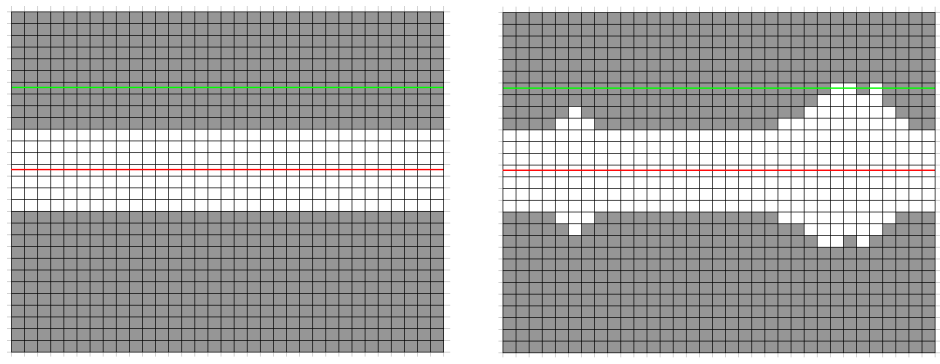

Figure 8: Two images demonstrating how the SICK ranger detects laser scatter details. The image on the left shows a laser not being scattered at all, so the pixels along the red line are never brightened while the image on the right shows two small scatter effects on the same laser, one of which is sufficient to brighten three of the pixels on the red line.

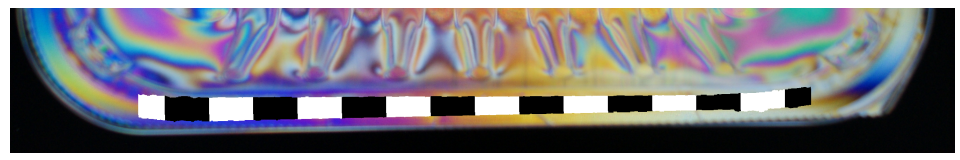

Figure 9: The classification regions for the seal in Figure 6.

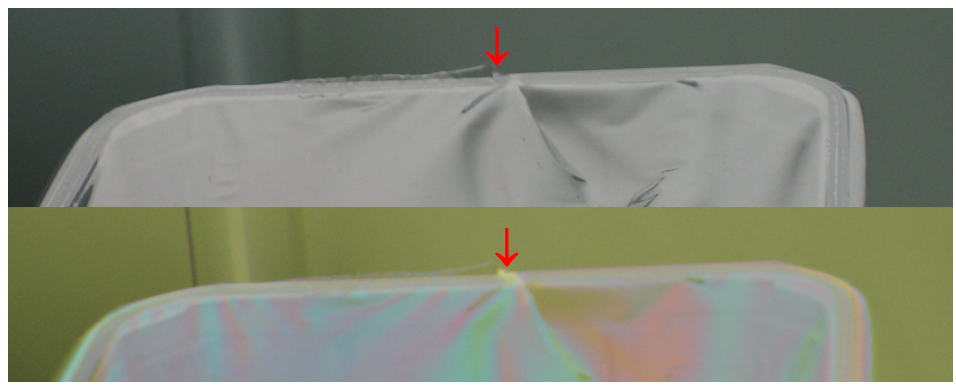

Figure 10: A pack with a sizable crease in the plastic film, highlighted under both plain light and a false-colour image comprised of three different polarisations of light. Intensity images for $0^{\circ}, 45^{\circ}$ and $90^{\circ}$ polarisations were used to provide the red, green and blue colour channels, respectively. While the fault is visible in both images, it is far more obvious in the seal region under the polarised image. 


\begin{tabular}{|l|c|c|c|}
\hline Filter type & Polarised & Laser & Combined \\
\hline \hline Raw & 0 & 5 & 0 \\
\hline Range & 2 & 9 & 0 \\
\hline Gradient & 3 & 17 & 3 \\
\hline Entropy (Raw) & 0 & 2 & 1 \\
\hline Entropy (Range) & 6 & 4 & 7 \\
\hline Entropy (Gradient) & 4 & 8 & 5 \\
\hline \hline Total & 15 & 45 & 16 \\
\hline
\end{tabular}

Table 1: The number of features selected from the polarised light, laser scatter and combined feature sets according to the filters described in Sections 2.4.1 to 2.4.3.

\begin{tabular}{|l|r|r|r|}
\hline Channel type & Polarised & Laser & Combined \\
\hline \hline red & 1 & - & 0 \\
\hline green & 3 & - & 1 \\
\hline blue & 4 & - & 3 \\
\hline $\max (\mathrm{R}, \mathrm{G}, \mathrm{B})$ & 3 & - & 2 \\
\hline $\min (\mathrm{R}, \mathrm{G}, \mathrm{B})$ & 2 & - & 2 \\
\hline range(R,G,B) & 1 & - & 4 \\
\hline intensity & 1 & - & 0 \\
\hline scatter & - & 17 & 2 \\
\hline direct & - & 28 & 2 \\
\hline \hline Total & 15 & 45 & 16 \\
\hline
\end{tabular}

Table 2: The number of features selected from the polarised light, laser scatter and combined feature sets according to the channels described in Sections 2.4.1 and 2.4.4. 


\begin{tabular}{|l|l|l|}
\hline Filter type & Channel type & Region statistic \\
\hline \hline Range entropy & intensity & mean \\
\hline Horizontal gradient entropy & red & $\max$ \\
\hline Range entropy & $\min (\mathrm{R}, \mathrm{G}, \mathrm{B})$ & skew \\
\hline Range entropy & $\max (\mathrm{R}, \mathrm{G}, \mathrm{B})$ & skew \\
\hline Range entropy & range $(\mathrm{R}, \mathrm{G}, \mathrm{B})$ & $\max$ \\
\hline Vertical gradient entropy & blue & mean \\
\hline Vertical gradient entropy & blue & var \\
\hline Horizontal gradient & green & mean \\
\hline Horizontal gradient & green & max \\
\hline Horizontal gradient & blue & mean \\
\hline Range entropy & $\min (\mathrm{R}, \mathrm{G}, \mathrm{B})$ & var \\
\hline Range entropy & $\max (\mathrm{R}, \mathrm{G}, \mathrm{B})$ & var \\
\hline Vertical gradient entropy & green & var \\
\hline Range & blue & min \\
\hline Range & $\max (\mathrm{R}, \mathrm{G}, \mathrm{B})$ & var \\
\hline
\end{tabular}

Table 3: The full list of features selected by AdaBoost for polarised light images. 


\begin{tabular}{|l|l|l|}
\hline Filter type & Channel type & Region statistic \\
\hline \hline Range entropy & $\min (\mathrm{R}, \mathrm{G}, \mathrm{B})$ & skew \\
\hline Range entropy & $\max (\mathrm{R}, \mathrm{G}, \mathrm{B})$ & skew \\
\hline Range entropy & $\operatorname{range}(\mathrm{R}, \mathrm{G}, \mathrm{B})$ & var \\
\hline Range entropy & range $(\mathrm{R}, \mathrm{G}, \mathrm{B})$ & $\max$ \\
\hline Vertical gradient entropy & blue & mean \\
\hline Vertical gradient entropy & blue & var \\
\hline Horizontal gradient & green & mean \\
\hline Horizontal gradient & blue & mean \\
\hline Range entropy & min(R,G,B $)$ & var \\
\hline Range entropy & max $(\mathrm{R}, \mathrm{G}, \mathrm{B})$ & var \\
\hline Range entropy & range $(\mathrm{R}, \mathrm{G}, \mathrm{B})$ & var \\
\hline Vertical gradient & range $(\mathrm{R}, \mathrm{G}, \mathrm{B})$ & min \\
\hline Horizontal gradient entropy & direct & mean \\
\hline Horizontal gradient entropy & direct & min \\
\hline Colour entropy & scatter & mean \\
\hline Gradient entropy & scatter & mean \\
\hline
\end{tabular}

Table 4: The full list of features selected by AdaBoost for the combination of laser scatter and polarised light images.

\begin{tabular}{|l|c|c|c|}
\hline & Sensitivity & Specificity & Accuracy \\
\hline \hline Polarised light & 0.96 & 0.96 & 0.96 \\
\hline Laser scatter & 0.88 & 0.90 & 0.90 \\
\hline Both & 0.94 & 0.96 & 0.95 \\
\hline
\end{tabular}

Table 5: The performance of the classifier using polarised light and/or laser scatter images. 